\title{
Os motivos que levam as pessoas idosas a recorrer frequentemente ao serviço de urgência
}

\author{
Alexandra Seara Almeida iD \\ Centro Hospitalar Universitário Lisboa Central - Pólo Hospital de Curry Cabral - Portugal \\ Idalina Delfina Gomes (iD \\ Escola Superior de Enfermagem de Lisboa - Lisboa - Portugal
}

\section{RESUMO}

Objetivo: Analisar os motivos da utilização frequente do serviço de urgência pelas pessoas idosas de uma unidade de saúde familiar. Métodos: Estudo descritivo qualitativo realizado em 2019 em uma unidade de saúde familiar no distrito de Lisboa, Portugal; a um total de 6 participantes (pessoas idosas frágeis e familiares cuidadores). A colheita de dados foi realizada por meio de entrevista semiestruturada e posteriormente analisada pela estratégia de análise de conteúdo. Resultados: Emergiram quatro categorias: Perceção de ser utilizador frequente do serviço de urgência; Serviços que dão apoio ao serviço de urgência; Situação de saúde / doença que levaram ao serviço de urgência; Razões que levaram a recorrer ao serviço de urgência. Verificou-se que a maioria dos motivos que levaram os participantes a recorrer ao serviço de urgência estavam relacionados com períodos de agudização da(s) doença(s) crónica(s) e as razões relacionadas com: a procura de cuidados especializados, referenciação por profissionais de saúde, medo de agravamento da situação de doença e dificuldade na acessibilidade à unidade de saúde. Conclusão: Os enfermeiros dos cuidados de saúde primários precisam de apostar mais na vigilância da(s) doenças crónicas(s) e na promoção da literacia da pessoa idosa e do familiar cuidador para promover o Cuidado-de-Si.

Descritores: Pessoa Idosa; Fragilidade; Utilizador Frequente; Serviço de Urgência.

\section{INTRODUÇÃO}

A medicina a par com a tecnologia tem vindo a evoluir, permitindo melhores condições sociais e de saúde levando ao aumento da esperança média de vida das pessoas quer a nível mundial, quer a nível nacional ${ }^{(1,2)}$.

O processo de envelhecimento é dinâmico, progressivo e influenciado por diversos fatores, nomeadamente: morfológicos, funcionais, bioquímicos, psicológicos, sociais, culturais, políticos e económicos. Quando estes fatores estão em desequilíbrio afetam a saúde, a capacidade funcional e a qualidade de vida da pessoa idosa, colocando-a mais predisposta à fragilidade ${ }^{(3)}$. Algumas destas alterações refletem-se em diferentes tipos de perdas: diminuição da capacidade em adaptar-se ao meio ambiente, limitações físicas, perdas cognitivas e sensoriais, perda da independência, perda do papel e posição social, isolamento social e sintomas depressivos ${ }^{(4)}$.

Por outro lado também o aumento da esperança média de vida da população reflete-se num aumento do número de pessoas idosas e num incremento de doenças crónicas como: diabetes, doenças cardíacas, hipertensão, doenças reumáticas, obesidade, demência, sendo cada vez mais raro morrer de doença aguda( ${ }^{(5)}$. O que faz com que seja necessário haver uma forte aposta na promoção da saúde. Essa aposta deverá incidir nos Cuidados de Saúde Primários (CSP), contudo, apesar dos esforços, continua-se a verificar uma crescente utilização do serviço de urgência $(S U)^{(6,7)}$. A definição do conceito utilizador frequente do serviço de urgência (UFSU) não é uma tarefa fácil e de consonância. Após uma análise da revisão bibliográfica verificou-se que a definição que reúne maior consenso é a que define UFSU os utentes que recorrem ao SU, num período de um ano um mínimo de quatro vezes ${ }^{(8,9)}$.

Assim têm-se vindo a desenvolver alguns estudos para perceber este problema que tem um forte impacto na saúde das populações. Um dos primeiros estudos realizados, sobre a utilização frequente do SU foi o estudo de James Mannon, em 1976 ${ }^{(10)}$. Desde então, a tomada de conhecimento para o aumento da utilização do SU tem sido uma realidade constante e frequente em vários países. Por exemplo, nos Estados Unidos da América, Canadá, Reino

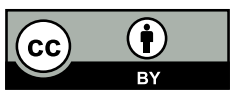

Este artigo está publicado em acesso aberto (Open Access) sob a licença Creative Commons, que permite uso, distribuição e reprodução em qualquer meio, sem restrições, desde que o trabalho seja corretamente citado. 
Unido e Austrália o aumento dos cuidados do SU é de $3 \%$ a $6 \%$ a cada ano. Mais especificamente, na Inglaterra os UFSU duplicaram de 6,8 milhões de utentes no período de 1966 a 1967, para 13,6 milhões no período de 2006 a 2007, e volta a aumentar para 14,3 milhões no período de 2012 a $2013^{(7)}$. De facto, os autores ${ }^{(6,7)}$ referem que esta tendência é confirmada na maioria dos países industrializados e Portugal não é exceção.

A utilização inadequada do SU contribui para o desperdício de recursos económicos, ameaçando a segurança e eficiência do SU e é responsável pelo elevado custo de exames não urgentes, levando a uma superlotação. Num estudo realizado em França ${ }^{(11)}$ verificou-se que o número de visitas ao SU aumentou $50 \%$ entre 2000 e 2015 . Outro estudo( $^{(6)}$ realizado na Carolina do Norte, em 2011, revela que as taxas de utilização do SU são 320 a $728 \%$ mais altas do que nos CSP, permitindo uma economia potencial de 69 a $86 \%$ se os utentes não urgentes fossem tratados em contextos de CSP em vez no SU. Outro estudo(12) realizado na Holanda, em 2016 , mostra que $41 \%$ a $52 \%$ dos episódios de urgência poderiam ser atendidos num serviço de CSP a custos mais baixos.

O aumento constante da utilização do SU nos últimos anos é uma fonte de pressão sobre os hospitais e o sistema de saúde levantando questões sobre a sua adequada utilização(12). O impacto deste aumento conduz à superlotação do SU, aumento de custos, diminuição da qualidade do serviço, aumento do tempo de espera, ambiente tenso devo a profissionais stressados e insatisfação do utente, o que leva à sobrecarga do serviço(6,7,12,13) afetando a sustentabilidade do sistema de saúde e economia ${ }^{(14)}$. Os fatores que levam ao aumento da utilização do SU são complexos e multifatoriais e prendem-se em grande parte, com o envelhecimento da população. Isto porque o aumento do número de pessoas com múltiplas condições crónicas torna-as mais vulneráveis e frágeis. Além disso, algumas das suas perspetivas relacionadas com os serviços de saúde contribuem igualmente para o aumento de episódios evitáveis (7,, $^{(13)}$.

Deste modo, os enfermeiros deverão estar preparados para avaliar e identificar as necessidades das pessoas idosas frágeis e agir de modo a controlar a fragilidade e os seus efeitos adversos ${ }^{(15)}$. O enfermeiro deverá estar igualmente preparado para estabelecer uma relação com a pessoa e familiar cuidador, em que os cuidados precisam abranger os domínios físico, ambiental e psicossocial, num modelo de intervenção em parceria com as pessoas idosas e familiares cuidadores para a promoção do Cuidado-de-Si ${ }^{(16)}$.

Conscientes desta problemática os enfermeiros da Unidade de Saúde Familiar "X" (USFX), e especialmente porque esta unidade apresentava uma taxa anual ajustada de episódios de urgência hospitalar de 62,39\%, perceberam a necessidade imperiosa de promover o Cuidado-de-Si às pessoas idosas, visando a prevenção e controlo da utilização frequente do SU, indo ao encontro das suas reais necessidades e potencialidades. O que passa necessariamente por compreender os motivos que levam as pessoas idosas e familiares cuidadores a serem UFSU. Na revisão da literatura efetuada, verificámos que são escassos os estudos qualitativos que incidem sobre os motivos da utilização frequente do SU sob ponto de vista das pessoas idosas e dos seus familiares cuidadores. Deste modo, pareceu-nos pertinente identificar os motivos da utilização frequente do SU pelas pessoas idosas da USFX.

Definimos como objetivo analisar os motivos da utilização frequente do SU pelas pessoas idosas. Como questão norteadora de investigação para o nosso estudo colocámos: Quais os motivos que levam as pessoas idosas da USFX a serem UFSU?

\section{MÉTODOS}

Recorreu-se à pesquisa qualitativa, uma vez que, está relacionada com a capacidade de compreensão do significado de um fenómeno e descrição pormenorizada dos contextos, sem dar relevância aos números "a quantidade é substituída pela intensidade, pela imersão profunda"(17) ou seja, não existe verdade absoluta, uma vez que se pretende estudar a complexidade de um fenómeno ${ }^{(18)}$. Para a colheita de dados recorreu-se à entrevista semiestruturada, porque pretendíamos obter informações sobre o que pensam os sujeitos do estudo ${ }^{(19)}$, ou seja perceber os motivos da utilização frequente do SU. A entrevista permite analisar dados fornecidos pelos entrevistados, referentes a conhecimento, a atitudes, a crenças e a sentimentos. Para realizar uma entrevista é necessário aplicar a arte de saber ouvir, saber perguntar e saber conversar. O entrevistador deverá saber ouvir de forma atenta e ativa, demonstrando interesse não apenas no que está a dizer o entrevistado, mas também nas suas emoções, de modo a compreender e aprofundar a conversa sem, contudo, influenciar a mesma ${ }^{(18)}$.

Para a análise dos dados obtidos recorremos à análise de conteúdo, isto é teve em conta os pressupostos teóricos da análise de conteúdo segundo Bardin (2016) cumprindo as três etapas: pré-análise, exploração do material e por último tratamento dos resultados, inferência e interpretação ${ }^{(20)}$. A pré-análise é uma fase em que se sistematizam as ideias iniciais, colocadas pelo quadro referencial teórico, de modo a elaborar um esquema compreensível que dê significado aos dados. Na exploração do material, codificam-se os dados, em que estes são transformados 
sistematicamente e agregados em unidades. Consiste na exploração do material com a definição de categorias e da identificação de unidades de registo. A categorização consiste na organização das categorias, onde estas e as subcategorias são explicadas, com base nas unidades de registo identificadas anteriormente. Ou seja, a categoria é uma forma de pensamento e reflete a realidade, de forma resumida, em determinados momentos ${ }^{(21)}$.

O estudo decorreu entre os dias quatro e treze de dezembro de 2019 e teve como população alvo: pessoas idosas utentes da USFX. Os participantes foram um total de três pessoas idosas e três familiares. A escolha dos participantes foi intencional, tendo em conta os critérios de inclusão nomeadamente: utentes da USFX; pessoas idosas com 65 e mais anos que após aplicação do instrumento de avaliação Mini Exame do Estado Mental não evidenciassem alterações cognitivas; pessoas idosas que fossem utilizadores frequentes SU; ou familiares cuidadores de pessoas idosas utentes da USFX que não fossem capazes de responder ${ }^{(22)}$.

Os participantes do estudo foram selecionados pelos enfermeiros de família, tendo em consideração os critérios de inclusão. O enfermeiro de família realizou os contatos a partir de consulta ou via telefone, abordando o entrevistado no sentido de o informar da existência do estudo, do objetivo do mesmo e da pertinência em participar. Após resposta positiva do utente, este era informado que seria contatado por uma das investigadoras. Esta abordagem permitiu diminui o fator surpresa, dar confiança ao entrevistado em participar diminuindo o seu possível nervosismo, de forma a que entrevista fosse o mais natural possível(17). Seguidamente, foi contatado o entrevistado para confirmar se continuava interessado em participar na entrevista. Novamente após confirmação positiva foi combinado o dia e local de acordo com a preferência do participante ${ }^{(22)}$.

No momento da entrevista a primeira abordagem consistiu em apresentar a investigadora e explicar a finalidade do presente estudo e o respetivo objetivo da entrevista. Foi igualmente explicado que a participação era voluntária, que seria assegurado o anonimato, que a entrevista seria audiogravada e que a qualquer momento poderia desistir, sem sofrer qualquer tipo de coação. Antes de se iniciar a entrevista foi permitido o esclarecimento de dúvidas. Após cumprir todos estes critérios, foi solicitado consentimento informado e esclarecido para dar início à entrevista. No decorrer da entrevista tentou-se proporcionar um ambiente cordial, um discurso adequado ao entrevistado, de modo a promover um ambiente harmonioso propício para o desenrolar da conversa e expressão de sentimentos e emoções. O local escolhido também facilitou neste sentido. O facto de ser o entrevistado a escolher o locar da entrevista (especialmente quando a opção foi o domicílio) possibilitou uma maior segurança e abertura de emoções e sentimentos. De referir que as entrevistas tiveram uma duração média de quinze minutos, uma vez que sendo pessoas idosas frágeis tivemos a preocupação de não os cansar demasiado. A entrevista foi terminada, igualmente, de forma cordial, agradecendo a participação, mostrando disponibilidade para possíveis esclarecimentos ${ }^{(22)}$.

Como foi referido, um dos critérios de inclusão consistia em identificar se existia alguma alteração do estado mental dos participantes, que comprometesse a informação fornecida pelos mesmos e quando assim fosse a entrevista era realizada aos familiares cuidadores. Deste modo, identificámos que dos seis participantes inicialmente referenciados três apresentaram alteração do estado mental e assim a entrevista foi realizada aos respetivos familiares cuidadores que demonstraram disponibilidade e desejo de participar ${ }^{(22)}$.

A transcrição das entrevistas é um passo deverás importante. Desta forma, foi realizada em ambiente silencioso, foram ouvidas inúmeras vezes e tentou-se ao máximo escrever na integra o conteúdo das mesmas, nomeadamente as pausas e a entoação da voz ${ }^{(22)}$.

Após a transcrição das entrevistas os textos foram lidos na integra linha a linha. Deste modo, realizou-se uma divisão manual da informação recolhida através das entrevistas em unidades manejáveis de síntese, na procura de padrões e de descoberta de aspetos importantes. Na categorização teve-se em conta os princípios da exclusividade entre categorias, da homogeneidade dentro das categorias e da objetividade entre os codificadores e a adequabilidade e pertinência aos conteúdos e objetivos ${ }^{(19)}$. O entrevistador identifica as categorias que podem ser a priori ou à posteriori, conforme a perspetiva da análise. No presente estudo, quer as categorias, quer as subcategorias foram definidas à posteriori, ou seja, de forma indutiva a partir dos significados referidos pelos participantes de forma explícita ${ }^{(17,21)}$. A análise da informação recolhida, foi agrupada em unidades manejáveis de síntese; em unidades de significação: em categorias, subcategorias e unidades de registo, na procura de padrões e de descoberta de aspetos importantes. De forma a simplificar as unidades de registo foram codificadas em "U" quando se referem a declarações prestadas pelos próprios utentes e em "F" quando as informações se referem aos familiares cuidadores. Para garantir a fiabilidade dos dados a análise de conteúdo foi aferida pelos dois investigadores ${ }^{(22)}$.

A realização do estudo obteve parecer favorável da Comissão de Ética para a Saúde para a ARSLVT - Proc.111/ CES/INV/2019. Todos os participantes foram informados sobre os objetivos do estudo e assinaram o consentimento informado. Garantiu-se o anonimato dos participantes e da instituição, bem como o sigilo e a confidencialidade das informações cedidas ${ }^{(22)}$. 


\section{RESULTADOS E DISCUSSÃO}

Dos seis entrevistados que participaram no estudo três eram pessoas idosas e os restantes três eram familiares cuidadores dos respetivos utentes. Em relação aos utentes, um era do sexo masculino e dois do sexo feminino. As idades estavam compreendidas entre os 70 e os 78 anos de idade. Após a aplicação do instrumento de avaliação da fragilidade Tilburg Frailty Indicator (TFI), pôde-se concluir que os três utentes entrevistados eram todos pessoas idosas frágeis. Relativamente aos familiares cuidadores dois destes eram os conjugues dos utentes e um deles era filha, dois eram do sexo feminino e um do sexo masculino. Todos viviam com o respetivo utente, eram igualmente utentes da USFX e após a aplicação da TFI, foram identificados como pessoas idosas frágeis ${ }^{(22)}$.

Da análise das entrevistas emergiram quatro categorias: Perceção de ser utilizador frequente do SU; Serviços que dão apoio ao SU, Situações de saúde/doença que levaram ao SU e Razões que levaram a recorrer ao SU e dez subcategorias ${ }^{(22)}$ como se pode constar no quadro que se segue:

Quadro I - Categorização das entrevistas.

\begin{tabular}{|ll|}
\hline CATEGORIAS & SUBCATEGORIAS \\
\hline Perceção de ser utilizador frequente do SU & Utilizador não frequente do SU \\
Serviços que dão apoio ao SU & Utilizador frequente do SU \\
& Conhecimento \\
Situações de saúde/doença que levaram ao SU & Desconhecimento \\
& Acidentes \\
& Agravamento da doença crónica \\
Razões que o levaram a recorrer ao SU & Procura de cuidados especializados \\
& Referenciado por profissionais de saúde \\
& Medo de agravamento da situação de doença \\
& Dificuldade na acessibilidade à USFX \\
\hline
\end{tabular}

Em relação à categoria "Perceção de ser utilizador frequente do SU", emergiram duas subcategorias: "Utilizador não frequente do SU" e "Utilizador frequente do SU". Conclui-se que apenas um dos seis participantes tinha a perceção de ser UFSU, como se pode constatar nos discursos dos familiares cuidadores:

"Foi quatro vezes, o que já são muitas vezes num ano." (F02)

Os restantes cinco participantes não tinham essa perceção, como elucidam os seguintes relatos:

"Só foi duas vezes." (F01)

"Fui poucas vezes, fui poucas vezes [...] Parece que fui uma vez ou duas." (U03)

A referir que após consentimento dos próprios, foi consultado o processo clínico, no sentido de confirmar se todos os utentes eram UFSU, tendo-se constado que sim (uma vez que este era um dos critérios de inclusão).

A partir da análise destes achados leva-nos a pressupor que os participantes podem não dar a devida importância quando recorrem ao SU. Por outro lado, podem ter esquecido do número de vezes que recorreram ao mesmo. O que nos leva a presumir que os participantes não têm a perceção de serem UFSU. Este achado revela-se de extrema importância, na medida em que, o enfermeiro deverá informar os utentes e familiares cuidadores sobre os vários serviços que dão apoio ao SU, essencialmente a Consulta Aberta Diária (CAD) da USFX e a linha de apoio telefónica "Saúde24". Deverá igualmente explicar a finalidade, funcionamento e modo de utilização de cada serviço e as implicações e consequências quando o SU é utilizado inadequadamente, portanto sem ser para situações urgentes, contribuindo para a utilização frequente do mesmo, levando-o à superlotação. Todos estes fatores levam ao aumento dos recursos, com aumento dos custos para a saúde, afetando a segurança e eficiência do SU e a própria sustentabilidade do sistema de saúde(6,7,12,13).

Relativamente à categoria "Serviços que dão apoio ao SU" resultaram as duas subcategorias: "Conhecimento" e "Desconhecimento". Após a análise das entrevistas, apenas um participante é que afirmou que conhecia que a USFX apresentava uma consulta que dava apoio ao SU, como nos referiu: 
"É só aqui. Já aqui venho há trinta anos, desde que tenho conhecimento desta consulta." (U04)

O participante estava a referir-se à CAD da USFX. Os outros participantes referiram:

"Não, não conheço. Não conheço outros serviços onde possa ir de urgência [...]" (F01)

"Não, não conheço. Não temos onde ir. É à urgência que eu sempre vou." (F02)

Esta dificuldade em identificar outros serviços que dão apoio ao SU, foi igualmente constatada na pesquisa efetuada, em que os autores ${ }^{(7,8,12,13)}$ afirmam que existe desconhecimento, por parte da população, sobre os diversos serviços que dão apoio ao SU.

Deste modo, evidencia-se, uma vez mais, a importância de os enfermeiros promoverem a educação e a informação. Deverão esclarecer e desmistificar os pré-conceitos que os utentes e os familiares cuidadores possuem relativamente o SU e sobre o desconhecimento da existência dos diversos serviços que dão apoio ao SU, especificamente neste contexto a CAD da USFX e a linha de apoio telefónica "Saúde24". Além disso, o enfermeiro deverá igualmente informar relativamente a cada serviço a sua finalidade, funcionamento e modo de utilização o que poderá evitar os episódios frequentes do SU e a incorreta utilização do mesmo. Como se consta em outro estudo(6) onde afirma que a utilização frequente do SU contribui para o desperdício de recursos económicos, ameaçando a segurança e eficiência do serviço. A utilização inadequada do SU é responsável pelo elevado custo de exames não urgentes, levando à sua superlotação.

Na categoria "Situações de saúde/doença que levaram ao SU" resultaram duas subcategorias: "Acidentes" e "Agravamento da doença crónica". Após a análise dos dados, constatou-se que a maior parte das situações de saúde que fizeram com que os participantes recorressem ao SU, estavam relacionados com períodos de agudização da doença(s) crónica(s), sobrepondo-se a situações associadas a acidentes, como se pode contatar do discurso destes participantes:

\section{"Ele ficou pior da doença que já tinha e tivemos que ir à urgência." (F02) \\ "Foi ao serviço de urgência porque piorou da sua doença crónica que tem [...]" (F04)}

Estes achados encontra-se em conformidade com outros estudos ${ }^{(6,7,9,13)}$ onde salientam que os motivos que levam ao aumento da utilização do SU são complexos e multifatoriais, prendendo-se com múltiplas condições crónicas, nomeadamente com aumento da fragilidade na pessoa idosa.

Assim, atualmente, os serviços de urgência têm revelado consciência sobre a tendência crescente de pessoas idosas que apresentam fragilidade. Estes serviços estão a evoluir de modo a que os cuidados agudos sejam mais direcionados para diminuir a fragilidade. A importância de identificar a fragilidade no SU está relacionada com a necessidade de compreender as manifestações agudas da doença, para prevenir eventos adversos durante e após a visita ao hospital, de forma a implementar intervenções destinadas a prevenir esses eventos e orientar os cuidados ${ }^{(15)}$.

Desta forma, uma vez que a pessoa idosa apresenta maior probabilidade de fragilidade, aquando da presença de doença aguda ou exacerbação da doença crónica, necessitando de cuidados específicos e complexos, é-lhe atribuído uma prioridade mais elevada comparativamente com os jovens adultos ${ }^{(9)}$. Contudo, muitos profissionais do SU apresentam uma especialização limitada em geriatria. Por conseguinte, esta falta de experiência pode levar a situações de: subtriagem, aumento das taxas de internamento, aumento do tempo de espera pelos cuidados, aumento do número de intervenções invasivas e desnecessárias levando a um maior tempo de permanência no SU' ${ }^{(23)}$.

As pessoas idosas, como UFSU, revelam ser uma população bastante heterogénea. A identificação das suas características permite melhorar a compreensão em relação às suas necessidades a nível dos cuidados de saúde e sociais, garantindo a qualidade de cuidados ${ }^{(9)}$. As diretrizes do SU geriátrico recomendam um atendimento multidisciplinar, um ambiente geriátrico-amigável com protocolos e indicadores de qualidade para rastrear a fragilidade ${ }^{(15)}$. O foco principal é oferecer atendimento de alta qualidade às pessoas idosas frágeis não só a nível do SU, mas essencialmente na continuidade de cuidados para os CSP. Deve-se incidir na articulação do SU com os CSP, apostando na acessibilidade aos CSP tendo em conta a particularidade da pessoa idosa frágil com doença crónica. Estas medidas poderão ter impacto na redução da utilização frequente do SU.

No presente estudo as situações de doença mais frequentes que levaram os utentes ao SU estavam relacionadas com o sistema circulatório e cardíaco, úlcera varicosa, úlcera diabética, dor, sistema urinário e respiratório. Como se pode constatar no discurso destes participantes:

"Úlcera varicosa na perna, na perna esquerda... No membro inferior esquerdo." (F02)

"A broncopneumonia, e falta de água no rim." (U04) 
Os motivos que mais se repetiram estavam relacionados com sintomas do foro cardíaco, como enunciam os seguintes relatos:

"As dores, as dores... ou tenho uma dor... ou apanha as vezes o lado do coração [...]" (U03)

"Um enfarte." (U05)

Estes achados vão ao encontro de outro estudo ${ }^{(9)}$ anteriormente realizado em que refere que a doença cardíaca é um dos motivos mais frequentes para ir ao SU.

Emergiu ainda que os participantes do presente estudo referiram que as situações de saúde que os fizeram recorrer ao SU da última vez, eram sempre os mesmos que das outras vezes:

"Foram, sempre os mesmos [...]" (U05)

Apenas um participante referiu que se dirigiu ao SU por motivos diferentes:

“Sim, motivos diferentes, foram sempre situações diferentes." (U04)

A análise leva-nos a pressupor que a(s) sua(s) doença(s) crónica(s) não se encontrava(m) bem controlada(s), o que chama a atenção para a importância do enfermeiro para a promoção do Cuidado-de-Si, tendo em conta o duplo sentido deste conceito: o de ajudar as pessoas idosas a gerir as situações de saúde doença, promovendo nelas o Cuidado-de-Si próprias ou capacitando os familiares cuidadores para assegurarem o cuidado do seu familiar doente ${ }^{(24)}$. Deste modo, quando o enfermeiro privilegia a educação/literacia está a permitir que o utente compreenda melhor os sinais e sintomas da(s) doença(s) crónica(s), conseguindo controlá-los mais eficazmente e quando surge um agravamento da sua saúde consiga escolher corretamente o serviço a que deve recorrer.

Por último na categoria "Razões que levaram a recorrer ao SU" identificaram-se quatro subcategorias. Após a análise dos dados, percebeu-se que por vezes os utentes recorrem ao SU devido à dificuldade na acessibilidade à USFX, como nos referiu este familiar cuidador:

"[...] porque no Centro de Saúde não me atendem na altura própria que preciso por isso tenho, tenho que ir para o hospital." (FO2)

Alguns utentes foram ao SU para terem acesso a cuidados especializados. Como nos referiu este participante:

"Quando, quando... a minha família pensa que o caso é para ir para São José, para a urgência de São José, eu vou para São José" (U03)

Outras vezes são mesmos os próprios profissionais de saúde (médico de família e assistentes em cuidados domiciliários) que os referenciam:

"E depois estava muito inchado e as senhoras que iam lá dar banho aconselharam-me a chamar o 112 para levar ao hospital." (F01)

"[...] quando o meu médico vê que eu não estou em condições." (U04)

Por outro lado, outros participantes consideraram que os sintomas precisavam de resposta urgente e referiram medo que os mesmos se agravem se não fosse atendido rapidamente como se pode constatar na seguinte frase:

"Bem eu só vou ao serviço de urgência quando efetivamente tenho mesmo medo que aconteça qualquer coisa mais grave." (F06).

Estes resultados vão ao encontro de outro estudo ${ }^{(7)}$, que revela que os utentes recorriam ao SU por considerem que a sua condição exigia recursos e instalações especializadas, por sentirem falta de confiança nos CSP, apresentarem desconhecimento de outros serviços de apoio e ainda por dificuldade no acesso aos CSP. Por outro lado, a opinião de familiares, amigos ou outros profissionais de saúde, também pesava na decisão de recorrer ao serviço de urgência, bem como o desejo de poderem ser atendidos rapidamente. Os autores ${ }^{(12)}$, salientaram ainda que algumas razões prendiam-se com medo de condições de risco de vida. Assim, torna-se primordial que os enfermeiros consigam desmitificar os pré-conceitos relacionados com o SU e sobre o funcionamento e atendimento dos CSP e no caso concreto deste estudo da USFX, mais especificamente a CAD e promovam um acompanhamento de proximidade com as pessoas idosas que as ajude a gerir as suas situações de saúde/fragilidade/doença no seu quotidiano de 
vida. Se os enfermeiros tiverem em consideração estes pressupostos estarão mais conscientes da importância de capacitar as pessoas idosas e os seus familiares cuidadores, de modo, a promover o seu empowerment, tendo por base uma teoria holística do cuidado num Modelo de cuidados centrado na pessoa( ${ }^{(25)}$.

\section{CONCLUSÃO}

Ao longo do tempo tem-se verificado um aumento crescente da utilização frequente do SU com aumento dos custos e sobrecarga do serviço. Após a análise das entrevistas realizadas aos seis participantes, pode-se constar que se tratavam todos de pessoas idosas frágeis em que a maioria não tinha a perceção que era um utilizador frequente do serviço de urgência. Este facto torna-se numa barreira para diminuir/controlar esta problemática.

Os participantes do estudo não identificaram a consulta aberta diária, inserida na USFX, como sendo um dos serviços que dá apoio ao SU para situações urgentes. Por outro lado, constatou-se que a maioria dos motivos que levaram os participantes a recorrer ao SU estavam relacionados com períodos de agudização da(s) doença(s) crónica(s), sobrepondo-se aos motivos associados a situações de doença aguda como por exemplo acidente, mais especificamente a quedas. As razões relacionadas com a procura de cuidados especializados; referenciação por profissionais de saúde; medo de agravamento da situação de doença e dificuldade na acessibilidade à USF forma aspetos que influenciaram a ida ao SU. O que realça a importância dos profissionais de saúde e nomeadamente dos enfermeiros dos CSP apostarem mais na prevenção e gestão da doença crónica e promoverem a literacia da pessoa idosa e do familiar cuidador em parceria, por forma a promover o Cuidado-de-Si, para um desenvolvimento sustentável da saúde, em que a saúde/doença seja vista como um projeto em inter-relação com o projeto de vida e existência da pessoa ${ }^{(26)}$. Além de ser necessário melhorar as condições do SU, tornando-o mais eficaz na prestação de cuidados as pessoas idosas, nomeadamente as mais frágeis. É também essencial continuar a realizar estudos no sentido de conhecer quem são os utilizadores frequentes, para posteriormente direcionar as políticas de saúde e gestão de cuidados para a melhoria destes serviços. Deste modo, a grande aposta precisa de incidir nos CSP, porque a falha de resposta destes serviços vai, inevitavelmente, aumentar a procura dos serviços de urgência.

O recurso a análise de conteúdo realizada focada no emergir dos significados que os participantes dão aos fenómenos quando estão em estudo problemas emergentes, mas ocultos, como o caso da utilização frequente do SU, contribui para mostrar a importância do desenvolvimento da pesquisa qualitativa.

Como limitação do estudo salienta-se o facto de não se ter conseguido incluir mais participantes, devido à dificuldade em identificar utentes como utilizadores frequentes do SU e as dificuldades colocadas pelas questões da pandemia. A salientar que como pertencemos à equipa de investigação do projeto a decorrer na USFX, a intenção é dar continuidade às entrevistas, contudo devido à situação pandémica, esta veio dificultar a continuação do projeto, porém retomaremos assim que seja oportuno.

\section{AGRADECIMENTOS}

Aos participantes do estudo e ao CIAIQ.

\section{CONFLITOS DE INTERESSE}

As autoras declaram a inexistência de conflitos de interesses na execução desta pesquisa.

\section{CONTRIBUIÇÕES}

Ambas as autoras contribuíram na elaboração e delineamento do estudo no seu todo. As autoras aprovaram a versão final enviada para publicação na Revista Brasileira em Promoção da Saúde (RBPS) e são responsáveis por todos aspectos do trabalho.

O presente artigo foi baseado no trabalho realizado no âmbito do Mestrado em Enfermagem Médico-Cirúrgica Pessoa Idosa na Escola Superior de Enfermagem de Lisboa intitulado: Prevenção da utilização frequente do serviço de urgência pelas pessoas idosas frágeis de uma Unidade de Saúde Familiar: Intervenção de enfermagem para a promoção do Cuidado- de- Si; Escola Superior de Enfermagem de Lisboa. A referir que o trabalho académico ainda não foi presente a discussão pública. Contempla um total de 220 páginas. 


\section{REFERÊNCIAS}

1. Gobbens RJ, Schols JM, Assen MA. Exploring the effciency of the Tilburg Frailty Indicator: a review. Clinical Interventions in Aging [Internet]. 2017;12:1739-1752. [Acesso em 2019 Nov 20]. Disponível em: http://www. zhga.nl/external/files/2017-10-19_R_Gobbens_TFI.pdf.

2. Carneiro $R$, Simões $C$, Carneiro $A H$. Plano individual e integrado de cuidados como objeto da relação clínica. Revista.SPMI [Internet]. 2019;26(2):147-152. [Acesso em 2019 Nov 20]. Disponível em: http://www.scielo. mec.pt/pdf/mint/v26n2/v26n2a13.pdf.

3. Kelly G, Mrengqwa L, Geffen L. "They don't care about us": older people's experiences of primary healthcare in Cape Town, South Africa. BMC Geriatrics [Internet]. 2019;19(98):1-14. [Acesso em 2019 Out 14]. Disponível em: https://doi.org/10.1186/s12877-019-1116-0.

4. Augusti ACV, Falsarella GR, Coimbra AMV. Análise da síndrome da fragilidade em idosos na atenção primária - Estudo transversal. Revista Brasileira de Medicina de Família e Comunidade, RBMFC [Internet]. 2017;12 (39):1-9. [Acesso em 2019 Out 14]. Disponível em: http://docs.bvsalud.org/biblioref/2018/03/848826/13538779-1-pb.pdf.

5. Observatório Português dos Sistemas de Saúde [OPSS]. Meio caminho andando - Relatório primavera 2018. OPSS [Internet]. 2018 [Acesso em 2019 Nov 20]. Disponível em: doi: opss.pt/wp-content/uploads/2018/06/ relatorio-primavera-2018.pdf.

6. Leporatti L, Ameri M, Trinchero C, Orcamo P, Montefiori M. Targeting frequent users of emergency departments: Prominent risk factors and policy implications. Health Policy [Internet]. 2016;120:462-470. [Acesso em 2019 Out 22]. Disponível em: https://www.ncbi.nlm.nih.gov/pubmed/27033015.

7. Coster JE, Turner JK, Bradbury D, Cantrell A. Why Do People Choose Emergency and Urgent Care Services? A Rapid Review Utilizing a Systematic Literature Search and Narrative Synthesis. Academic Emergency Medicine [Internet]. 2017;24(9):1137-11-49. [Acesso em 2019 Out 22]. Disponível em: https://www.ncbi.nlm. nih.gov/pmc/articles/PMC5599959/pdf/ACEM-24-1137.pdf.

8. Legramante JM, Morciano L, Lucaroni F, Gilardi F, Caredda E, Pesaresi A, et al. Frequent use of Emergency Departments by the elderly population when continuing care - Is not well established. PLOS ONE [Internet]. 2016;11(12):1-11. [Acesso em 2019 Out 20]. Disponível em: doi: 10.1371/journal.pone.0165939.

9. Dufour I, Chouinard MC, Dubuc N, Beaudin J, Lafontaine S, Hudon C. Factors associated with frequent use of emergency-department services in a geriatric population: a systematic review. BMC Geriatrics [Internet]. 2019;19(185):1-9. [Acesso em 2019 Out 20]. Disponível em: https://bmcgeriatr.biomedcentral.com/track/ $\mathrm{pdf} / 10.1186 / \mathrm{s} 12877-019-1197-9$.

10. Burns T R. Contributing factors of frequent use of the emergency department: A synthesis. International Emergency Nursing [Internet]. 2017:1-5. [Acesso em 2019 Out 23]. Disponível em: http://dx.doi.org/10.1016/j. ienj.2017.06.001.

11. Or Z, Penneau A. A Multilevel Analysis of the determinants of emergency care visits bythe elderly in France. Health Policy [Internet]. 2018:1-7. [Acesso em 2019 Out 20]. Disponível em: https://doi.org/10.1016/j. healthpol.2018.05.003.

12. Kraaijvanger N, Rijpsma D, Willink L, Lucassen $P$, Leeuwen $H$, Edwards $M$. Why patients self-refer to the Emergency Department: A qualitative interview study. Journal of Evaluation in Clinical Practice [Internet]. 2016;23:593-598. [Acesso em 2019 Out 22]. Disponível em: https://bmcgeriatr.biomedcentral.com/track/ pdf/10.1186/s12877-019-1197-9. https://onlinelibrary.wiley.com/doi/epdf/10.1111/jep.12680.

13. Andrews $\mathrm{H}$, Kass L. Non-urgent use of emergency departments: populations most likely to overestimate illness severity. Internal and Emergency Medicine [Internet]. 2018:1-8. [Acesso em 2019 Out 20]. Disponível em: https://www.ncbi.nlm.nih.gov/pubmed/29380133.

14. Kuek BJW, Li H, Yap S, Ng MXR, Ng YY, White AE, et al. Characteristics of frequent users of Emergency Medical Services in Singapore. PREHOSPITAL EMERGENCY CARE [Internet]. 2018:215-224. [Acesso em 2019 Out 20]. Disponível em: https://doi.org/10.1080/10903127.2018.1484969. 
15. Hoogendijk EO, Afilalo J, Ensrud KE, Kowal P, Onder G, Fried LP. Frailty: implications for clinical practice and public health. The Lancet [Internet]. 2019;394:1365-1375. [Acesso em 2019 Set 12]. Disponível em: https:// doi.org/10.1016/S0140-6736(19)31786-6.

16. Gomes ID. Promover o cuidado de si: parceria entre o enfermeiro e a pessoa idosa. A construção do processo de parceria num contexto de vulnerabilidade e dependência. $1^{\text {a }}$ ed. Novas Edições Académcias; 2016.

17. Taquette SR. Análise de dados de pesquisa qualitativa em saúde. Atas CIAIQ 2016 [Internet]. 2016;2:524533. [Acesso em 2019 Set 11]. Disponível em: doi: proceedings.ciaiq.org/index.php/ciaiq2016/article/ view/790.

18. Batista $E C$, Matos $L A L$, Nascimento $A B$. A entrevista como técnica de investigação na pesquisa qualitativa. Revista Interdisciplinar Científica Aplicada [Internet]. 2017;11(3):23-38. [Acesso em 2019 Set 11]. Disponível em: file:///C:/Users/EU/Downloads/Aentrevistacomotcnicadeinvestigaonapesquisaqualitativa.pdf.

19. Minayo MCS, Costa AP. Fundamentos teóricos das técnicas de investigação qualitativa. Revista Lusófona de Educação [Internet]. 2018;40:139-153. [Acesso em 2019 Set 11]. Disponível em: https://revistas.ulusofona.pt/ index.php/rleducacao/article/view/6439.

20. Bardin L. Análise de conteúdo. $1^{\text {a }}$ ed. Lisboa: Edições 70; 2016.

21. Leite RF. Perspectiva da análise de conteúdo na pesquisa qualitativa: algumas considerações. Revista Pesquisa Qualitativa [Internet]. 2017;5(9):539-551. [Acesso em 2019 Set 11]. Disponível em: doi: editora. sepq.org.br/rpq/article/view/129/98.

22. Gomes ID, Almeida AS. Motivos da utilização frequente do serviço de urgência pelas pessoas idosas no contexto de uma unidade de saúde familiar. Em: Fornari L, Oliveira ESF, Oliveira C, Faria BM, Ribeiro J, Higa EFR, editores. Investigação Qualitativa em Saúde: Avanços e desafios. New trends in Qualitative Research. Ludomedia. Portugal. 2021;8:600-605. [Acesso em 2021 Ago 13]. Disponível em: doi: https://doi. org/10.36367/ntqr.8.2021.600-605.

23. Marsden E, Taylor A, Wallis M, Craswell A, Broadbent M, Barnett A, et al. A structure, process and outcome evaluation of the Geriatric Emergency Department Intervention model of care: a study protocol. BMC Geriatrics [Internet]. 2017;17(76):1-8. [Acesso em 2019 Out 14]. Obtido de https://www.ncbi.nlm.nih.gov/pmc/ articles/PMC5363028/pdf/12877_2017_Article_462.pdf.

24. Gomes I. Partnership of Care in the Promotion of the Care-of-the-Self: An Implementation Guide with Elderly People. Em: Garcia-Alonso J, Fonseca C, editores. Gerontechnology III: Contributions to the Third International Workshop on Gerontechnology, IWoG 2020, Portugal. Évora: Lecture Notes in Bioengineering: Springer; 2021. p. 345-356.

25. McCance T, McCormack B, Slater P, McConnell D. Examining the theoretical relationships between constructs in the person-centered practice framework: a structural equation model. BMC Health Services Research [Internet]. 2020:1-23. [Acesso em 2020 Set 29]. Disponível em: doi: 10.21203/rs.3.rs-22586/v1.

26. Gomes ID. Promover o Cuidado-de-Si: património da enfermagem para o desenvolvimento sustentado, bemestar e saúde das populações. Revista Pensar Enfermagem. 2019;23(2):7-15.

\author{
Endereço para correspondência: \\ Alexandra Seara Almeida \\ Travessa do Mocho, n. ${ }^{\circ} 2,1^{\circ}$ dto \\ Código Postal: 2665-605 - Venda do Pinheiro - Mafra - Portugal \\ E-mail: alexandraseara51@gmail.coms
}

